\title{
V7. DIETARY RETINOL ROLE AND RETINOIC ACID ADJUVANT EFFECT IN MUCOSAL LaAg VACCINE EFFICACY AGAINST Leishmania amazonensis INFECTION.
}

Izabella Pereira da Silva Bezerra ${ }^{1}$; Júlia Gama de Azevedo ${ }^{1}$; Bartira Rossi Bergmann ${ }^{1}$.

${ }^{1}$ Instituto de Biofísica Carlos Chagas Filho, Universidade Federal do Rio de Janeiro.

INTRODUCTION Leishmaniases are diseases that have different clinical manifestations depending on the parasite species involved and on the host immune status. Despite the intense effort in finding a safe and effective vaccine against leishmaniases, none has been approved yet for human use. Our group has been exploring a new vaccination strategy through the mucosa to induce tolerance to parasite antigens related to the disease. We have already demonstrated that oral and intranasal immunization with total antigen of L. amazonensis promastigotes ( $\mathrm{LaAg}$ ) induces protection in mice against cutaneous leishmaniasis. It is well established that mucosas are involved with immune tolerance induction, given that they are in constant contact with exogenous antigens. Retinoic acid (RA), a metabolite of retinol (vitamin A), is a cofactor required for generation of regulatory $\mathrm{T}$ cells in the intestinal mucosa, which have been associated with immune tolerance.

OBJECTIVE In this work, the influence of dietary retinol in the efficacy of oral and intranasal $\mathrm{LaAg}$ vaccines was evaluated. We also evaluated the adjuvant role of retinoic acid in intranasal $\mathrm{LaAg}$ vaccine efficacy.

METHODOLOGY BALB/c mice subjected to dietary retinol restriction (Retinol ${ }^{-}$) or supplementation $\left(\right.$Retinol $\left.^{+}\right)$were orally $(100 \mu \mathrm{g}$ of protein/dose) or intranasally (10 $\mu \mathrm{g}$ of protein/dose) vaccinated with two doses of $\mathrm{LaAg}$ with a seven day interval between them. One week after immunization, mice were subcutaneously infected in the footpad with $L$. amazonensis promastigotes and the lesion development was monitored for 60 days, when the parasite burden and cytokine profile in the infection site were evaluated. To evaluate the adjuvant role of RA, we used RA encapsulated in solid lipid nanoparticles (RA-SLN, $15 \mu \mathrm{g}$ of RA/dose), which was administered with the $\mathrm{LaAg}$ intranasal vaccine in normally fed $\mathrm{BALB} / \mathrm{c}$ mice. 
RESULTS Retinol ${ }^{-}$mice were more resistant to infection than Retinol+ mice, presenting a lower lesion development and a reduced parasite burden. However, oral or intranasal $\mathrm{LaAg}$ vaccine were only protected by Retinol+ mice, which presented increased IL-12 and reduced IL-4 levels in the lesions compared to non-vaccinated animals. Moreover, Retinol- mice presented reduced TGF- $\beta$ levels in the lesions compared to Retinol ${ }^{+}$animals. We also observed that RA-SLN increased LaAg intranasal vaccine efficacy and enhanced $\mathrm{CD} 4^{+} \mathrm{Foxp}^{+}$population in cervical lymph nodes after immunization.

CONCLUSION Altogether, these results show that dietary retinol was required for both oral and intranasal $\mathrm{LaAg}$ vaccine efficacy against $L$. amazonensis infection. Moreover, RA-SLN was a good adjuvant for $\mathrm{LaAg}$ intranasal vaccine, inducing regulatory $\mathrm{T}$ cell generation in draining lymph nodes. These data suggest a tolerogenic effect of mucosal $\mathrm{LaAg}$ vaccine.

KEYWORDS leishmania, LaAg, vaccine, oral, intranasal, retinol, retinoic acid, immune tolerance. 